\title{
Utilità e costo/efficacia del dinoprostone in ostetricia
}

Mario Eandi ${ }^{\circ}$, Lorenzo Pradelli ${ }^{\circ}$, Chiara Benedetto*

PROFILI

\begin{abstract}
Pregnancy-related hypertension, intrauterine growth retardation, post-maturity and unfavorable local conditions are among the most frequent indications for labor induction. There are two competing strategies for the induction of labor in term pregnancies: formal induction with artificial rupture of the membranes and/or intravenous oxytocin on one side, and cervical ripening and induction with exogenous prostaglandins on the other.

The use of prostaglandins in obstetrics relies on two pharmacological properties: the capacity of inducing the biochemical changes in the connective tissue of the cervix that lead to its maturation and the stimulation of the uterine smooth musculature. While the latter property is shared with oxytocin, the former offers great clinical advantages, in particular in those women that present an indication for labor induction but whose cervix, normally assessed with the use of the Bishop score, does not show "ripeness", i.e. does not permit vaginal delivery. Since the acquisition cost of the prostaglandins is quite high, but the clinical benefit appears evident, several studies have analyzed the overall economical impact of their use, both as pre-induction cervical ripening agents and as induction drugs.

When compared to a strategy of expectant management in term pregnancies without cervical ripeness, exogenous prostaglandin administration has proven to be more cost-effective and better accepted by the patients.

Prostaglandins have also proven to be more cost-effective than oxytocin and/or amniotomy in women with an indication to labor induction and unripe cervix, due to the reduced number of induction failures and cesarean sections required with this strategy. The economical analyses that have compared oxytocin and prostaglandins in women with ripe cervices deliver more ambiguous data, but it appears that their cost-effectiveness is comparable. Furthermore, none of the reviewed studies has considered intangible costs, but it is a wide-spread opinion that induction with prostaglandins results in a more "natural" and less painful labor.
\end{abstract}

Farmeconomia e percorsi terapeutici 2002; 3 (3): 135 - 146

\section{INTRODUZIONE}

Le indicazioni all'induzione del travaglio di parto nelle donne con gravidanza a termine o in prossimità di esso sono molteplici, di ordine sia medico sia ostetrico, e comprendono motivi materni e/o fetali, tra cui l'ipertensione in gravidanza, il ritardo di crescita intrauterino, la gravidanza di elevata età gestazionale, la nefropatia e il diabete materni, la pre-eclampsia e la rottura pretravaglio delle membrane. $\mathrm{La}$ causa primaria per cui si ricorre all'induzione del travaglio è il tentativo di evitare il parto cesareo (1).

Per molti anni la procedura standard per l'induzione del travaglio di parto si è basata sull' amnioressi (rottura artificiale delle membrane) e sulla somministrazione endovenosa di ossitocina. Negli ultimi anni, a questa procedura classica si è affiancata una strategia alternativa, consistente nella somministrazione di prostaglandine esogene per favorire la maturazione del collo dell'utero e indurre il travaglio di parto.

Di fronte a una gestante con una possibile indicazione all' induzione del travaglio di parto, in particolare se la cervice uterina si presenta immatura, il medico ostetrico può dunque scegliere tra varie alternative terapeutiche ed in particolare deve decidere se sia preferibile attendere la maturazione spontanea della cervice e l'inizio del travaglio oppure assumere un atteggiamento più interventista, somministrando prostaglandine o inducendo il travaglio con la procedura classica. Qualora abbia scelto di somministrare le prostaglandine
${ }^{\circ}$ Farmacologia Clinica, Università di Torino

* Direttore della Cattedra C di Ginecologia e Ostetricia, Università di Torino, Azienda Ospedaliera OIRM-S.Anna 
per favorire la maturazione, deve decidere quanto tempo é opportuno aspettare, o quante volte é lecito ripetere la loro somministrazione prima di indurre il travaglio. Inoltre, deve decidere quale tecnica di induzione sia piú vantaggiosa se il travaglio non é insorto durante la fase di maturazione cervicale.

Obiettivo di questo lavoro é analizzare le implicazioni cliniche ed economiche di queste scelte alla luce delle valutazioni farmacoeconomiche pubblicate sull'utilizzo delle prostaglandine (dinoprostone) in ostetricia.

\section{Definizione del problema}

L'utilizzo clinico delle prostaglandine esogene in ostetricia si basa sulla loro doppia capacità di indurre la maturazione della cervice uterina (2) e di stimolare le contrazioni del miometrio $(3,4)$. Attualmente, in Italia, l'unica prostaglandina registrata per queste indicazioni è il dinoprostone, o prostaglandina E2, disponibile come gel per applicazione intracervicale $(0,5 \mathrm{mg})$ o endovaginale $(1 \mathrm{mg}$ e $2 \mathrm{mg}$ ) e come pessario a rilascio prolungato.

I prodotti destinati alla somministrazione intracervicale si differenziano da quelli per l'applicazione vaginale non solo per il dosaggio, ma anche per la differente indicazione, essendo i primi indicati soprattutto per la maturazione cervicale pre-induzione, e i secondi per la maturazione cervicale e l'induzione del travaglio vera e propria.

Sia l'ossitocina che le prostaglandine hanno la capacità di stimolare l'insorgenza delle contrazioni del miometrio che caratterizzano il travaglio di parto, ma queste ultime hanno una caratteristica farmacologica aggiuntiva, che conferisce loro un vantaggio nell'induzione del travaglio in donne con maturazione cervicale incompleta: la capacità di indurre modificazioni dell'attività collagenasica all'interno del collo dell'utero (5). Infatti, una delle principali cause di fallimento dell'induzione, qualunque sia la tecnica utilizzata, consiste nell' "immaturità" della cervice uterina: in questa condizione, l'insorgenza di contrazioni uterine, per cause sia fisiologiche sia iatrogene, non è sufficiente all'espulsione del feto, che, anzi, viene esposto ad uno stress meccanico inutile.

La tecnica tradizionale di induzione del travaglio, basata sull'amnioressi e sull'ossitocina, è in grado solamente di stimolare le contrazioni del miometrio, mentre non ha alcun effetto sulla maturazione cervicale, indispensabile per consentire il passaggio del feto lungo il canale del parto. Com'è noto, infatti, per permettere il passaggio del nascituro la cervice deve subire un rimodellamento, l'appianamento, regolato da una serie di modificazioni biochimiche che portano ad un "rammollimento" del tessuto connettivo, che consente la dilatazione del canale cervicale.

Somministrate per via intracervicale, le prostaglandine esogene inducono il rammollimento del collo dell'utero, nella maggior parte dei casi dopo 6-12 ore. Quest'effetto può associarsi o meno alla stimolazione dell'attività contrattile uterina.

Il parametro di valutazione della maturazione cervicale più utilizzato e validato è lo score di Bishop, un sistema a punteggio proposto nel 1964 (6) per la valutazione del grado di maturazione cervicale sulla base dei reperti rilevati all'esame obiettivo ginecologico. Tale score si è rivelato il singolo miglior predittore del successo dell'induzione del travaglio di parto.

\section{VALUTAZIONI FARMACOECONOMICHE}

La gravidanza di elevata età gestazionale (> 41 settimane) costituisce una frequente situazione clinica, in cui la prima scelta che si trova a dover affrontare il medico é quella tra un atteggiamento conservativo, di "vigile attesa", e uno più aggressivo, con induzione del travaglio di parto.

Uno studio canadese di Goeree et al. del 1995 (7) ha condotto un' analisi di costo-efficacia comparata tra queste due strategie alternative, basandosi sui dati forniti da un ampio studio clinico controllato, il Canadian Multicentre Post-Term Pregnancy Trial. Tale studio ha arruolato 3407 donne con gravidanza non complicata oltre le 41 settimane, suddividendole in due gruppi randomizzati: il primo é stato assegnato ad una gestione conservativa, che prevedeva il monitoraggio seriato delle condizioni fetali e materne in attesa dell'insorgenza spontanea del parto; le donne assegnate al secondo gruppo, invece, sono state indotte con gel di dinoprostone somministrato per via intracervicale, fino a un massimo di tre dosi da $0,5 \mathrm{mg}$, eventualmente seguite da amnioressi e/o ossitocina. Non sono emerse differenze significative tra i due gruppi per quanto riguarda i principali outcomes clinici, che erano la mortalità perinatale e la morbilità neonatale, mentre l'atteggiamento interventista é risultato associato ad una riduzione significativa $(\mathrm{p}<0,03)$ del tasso di tagli cesarei.

Poiché le principali misure d'efficacia erano risultate non significativamente diverse tra i due gruppi, gli autori dell' analisi economica hanno adottato la tecnica "minimizzazione dei costi", considerando il tasso di tagli cesarei 
un fattore di costo, piuttosto che una misura d'efficacia clinica.

L'analisi minimizzazione dei costi é stata condotta nella prospettiva del Servizio Sanitario dell'Ontario. I tipi di costo presi in considerazione comprendevano quelli ospedalieri, farmaceutici e le tariffe professionali.

I risultati dell' analisi, espressi come costo medio per paziente riferiti ai due gruppi di studio (tabella 1), indicano che la strategia che prevedeva l'induzione del travaglio era risultata mediamente meno cara di 193 Dollari Canadesi (valore del 1992); questa differenza era dovuta principalmente al minor ricorso ai test necessari al monitoraggio seriale prenatale e alla minor probabilità di dover ricorrere al taglio cesareo dopo la procedura di induzione.

Sulla base di queste differenze di costo, gli autori hanno stimato i risparmi potenziali su base nazionale associati all'adozione universale di una politica di induzione del travaglio nelle gestanti di elevata età gestazionale, assumendo che in Canada queste rappresentino il $10 \%$ del totale. Dopo aver bilanciato i risultati per compensare l'eccessiva rappresentanza di ospedali universitari e di gestanti primipare nel loro campione, gli autori hanno calcolato un risparmio annuo pari a 8 milioni di Dollari Canadesi per anno. Nell' articolo viene sottolineato che, nonostante la differenza di costo sia di per sé significativa, l'analisi non ha preso in considerazione i costi e la qualità di vita della paziente, e che pertanto il risparmio reale é stato presumibilmente sottostimato. Secondo gli autori, la gestione delle gravidanze di elevata età gestazionale che prevede l'induzione con dinoprostone, eventualmente seguita dall'induzione formale con amnioressi e/o ossitocina, risulta dominante rispetto alla strategia alternativa di "vigile attesa", in quanto produce esiti clinici migliori ad un costo inferiore.

Un'altra situazione clinica frequente in cui le possibili alternative sono rappresentate dall'induzione del travaglio e dalla gestione conservativa, con attesa della sua insorgenza spontanea, é costituita dalla rottura pre-travaglio delle membrane in gestanti a termine $(>37$ settimane).
In questi casi, alcuni clinici preferiscono indurre il travaglio il prima possibile, per timore che una lunga latenza tra la rottura delle membrane e l'insorgenza del travaglio si associ ad un aumentato rischio di infezioni neonatali, mentre altri ritengono piú appropriato attendere l'insorgenza spontanea del travaglio, giudicando che tale atteggiamento si associ a una probabilità ridotta di parto cesareo. Per verificare queste ipotesi, é stato condotto un ampio studio multicentrico (8) che ha coinvolto 5041 pazienti con gravidanza a termine afferenti a 72 ospedali situati in Canada, Gran Bretagna, Australia, Israele, Svezia e Danimarca, denominato TERMPROM (Term Prelabour Rupture of the Membranes).

Dopo l'inclusione nello studio, le donne arruolate sono state randomizzate in quattro gruppi; le donne dei primi due gruppi sono state sottoposte ad induzione immediata con prostaglandine (iPG) o con ossitocina (iOT), mentre le pazienti degli altri due gruppi sono state gestite in maniera conservativa, attendendo l'insorgenza spontanea del travaglio, che veniva indotto artificialmente con prostaglandine (aPG) od ossitocina (aOT) solo in caso di insorgenza di complicazioni. Lo studio non ha rilevato alcuna differenza statisticamente significativa nei tassi di infezioni neonatali e di tagli cesarei tra i quattro gruppi di trattamento, mentre ha rilevato un vantaggio della strategia iOT in termini di infezioni materne e di tempo intercorso tra l'inizio della presa in carico della paziente e il parto. Un questionario somministrato alle gestanti ha inoltre evidenziato che le pazienti incluse nei gruppi sottoposti ad induzione immediata erano significativamente più soddisfatte del loro trattamento di quelle gestite in maniera conservativa. Lo studio TERMPROM comprendeva anche una valutazione economica (9) delle quattro strategie alternative considerate; l'analisi è stata condotta assumendo la prospettiva dei terzi paganti delle tre nazioni che avevano contribuito maggiormente allo studio in termini numerici, cioè Canada, Gran Bretagna e Australia. In accordo con la prospettiva assunta, i costi presi in considerazione comprendevano tutti i costi di trattamento per la madre ed il neonato, cioè quelli

\begin{tabular}{|lcccc|}
\hline & \multicolumn{2}{c}{ Gruppo di studio } & \multicolumn{2}{c|}{ Differenza tra } \\
Categoria di costo & Monitoraggio & Induzione & gruppi (I.C. 95\%) & P \\
\hline Costi ospedalieri & 2684 & 2502 & $182(129-234)$ & $<0,0001$ \\
\hline Tariffe professionali & 448 & 437 & $11(1-21)$ & 0,025 \\
\hline Totale & 3132 & 2939 & $193(133-252)$ & $<0,0001$ \\
\hline
\end{tabular}

Modificata da Goeree et al (7)

Tabella 1

Costi (C\$) medi per paziente con gravidanza di elevata età gestazionale nelle due strategie alternative 


\begin{tabular}{|l|cccc|ccc|}
\hline & \multicolumn{4}{|c|}{ Strategia adottata } & \multicolumn{3}{c|}{ Differenze inter-gruppo } \\
\hline Nazione & iOT & iPG & aOT & aPG & iOT vs. aOT (p) & iPG vs. aPG (p) & iOT vs. iPG (p) \\
\hline Canada (C\$) & 3056 & 3102 & 3170 & 3142 & $-114(0,004)$ & $-40(0,9321)$ & $-46(0,0217)$ \\
\hline Gran Bretagna (£) & 1110 & 1173 & 1223 & 1203 & $-113(0,0001)$ & $-30(0,368)$ & $-63(0,0004)$ \\
\hline Australia (A\$) & 2191 & 2240 & 2221 & 2237 & $-30(0,0342)$ & $3(0,4369)$ & $-49(0,0084)$ \\
\hline
\end{tabular}

$C \$=$ Dollari Canadesi, $£=$ Sterline Inglesi, $A \$=$ Dollari Australiani. Modificata da Gafni et al (9).

Tabella 2

Costi medi per paziente per strategia terapeutica e differenze inter-gruppo

ospedalieri, le tariffe professionali e la spesa per l'acquisto dei farmaci utilizzati per l'induzione, mentre non erano valutati i costi per il paziente, né quelli secondari alla perdita di produttività. I risultati dell' analisi di costo (tabella 2) indicano che la strategia "interventista" risultava sempre essere maggiormente conveniente: la procedura iOT era quella meno cara e l'aPG quella più costosa, sebbene la differenza di costo tra le strategie alternative variasse sensibilmente a seconda del Paese considerato.

Nel commentare i loro risultati, Gafni e coll. rimarcavano il fatto che le differenze di costo stimate erano statisticamente significative, ma che ciò non implicava che avessero una valenza pratica ugualmente significativa, costituendo solo una piccola percentuale del costo complessivo del trattamento. Essi ritengono che entrambe le strategie siano giustificabili dal punto di vista clinico ed economico, almeno nei paesi più sviluppati, e che pertanto la scelta dovrebbe essere affidata alla paziente, pienamente informata, in quanto da un lato con la strategia dell'induzione immediata si hanno minori probabilità di incorrere in infezioni materne post-partum, dall'altro alcune pazienti presentano un'avversione all'induzione del travaglio.

Una valutazione farmacoeconomica sull'utilizzo del dinoprostone, sotto forma di gel, è stata condotta negli USA da Hass e Lucas (10), adottando i parametri economici relativi a quel Paese, riferiti al 1993. In questo lavoro, l'attenzione si è concentrata sull'utilizzo del dinoprostone per favorire la maturazione della cervice, senza considerare il suo utilizzo nell'induzione del travaglio vera e propria.

La tecnica di analisi impiegata si basa sulla costruzione di un albero decisionale, in cui ad ogni alternativa terapeutica vengono associate le possibili conseguenze, corredate dai rispettivi costi e probabilità. L'albero decisiona-

\section{Figura 1}

Albero decisionale per la gestione dell'induzione del travaglio in gestanti con cervice immatura.

Tratto da Hass e Lucas (10)

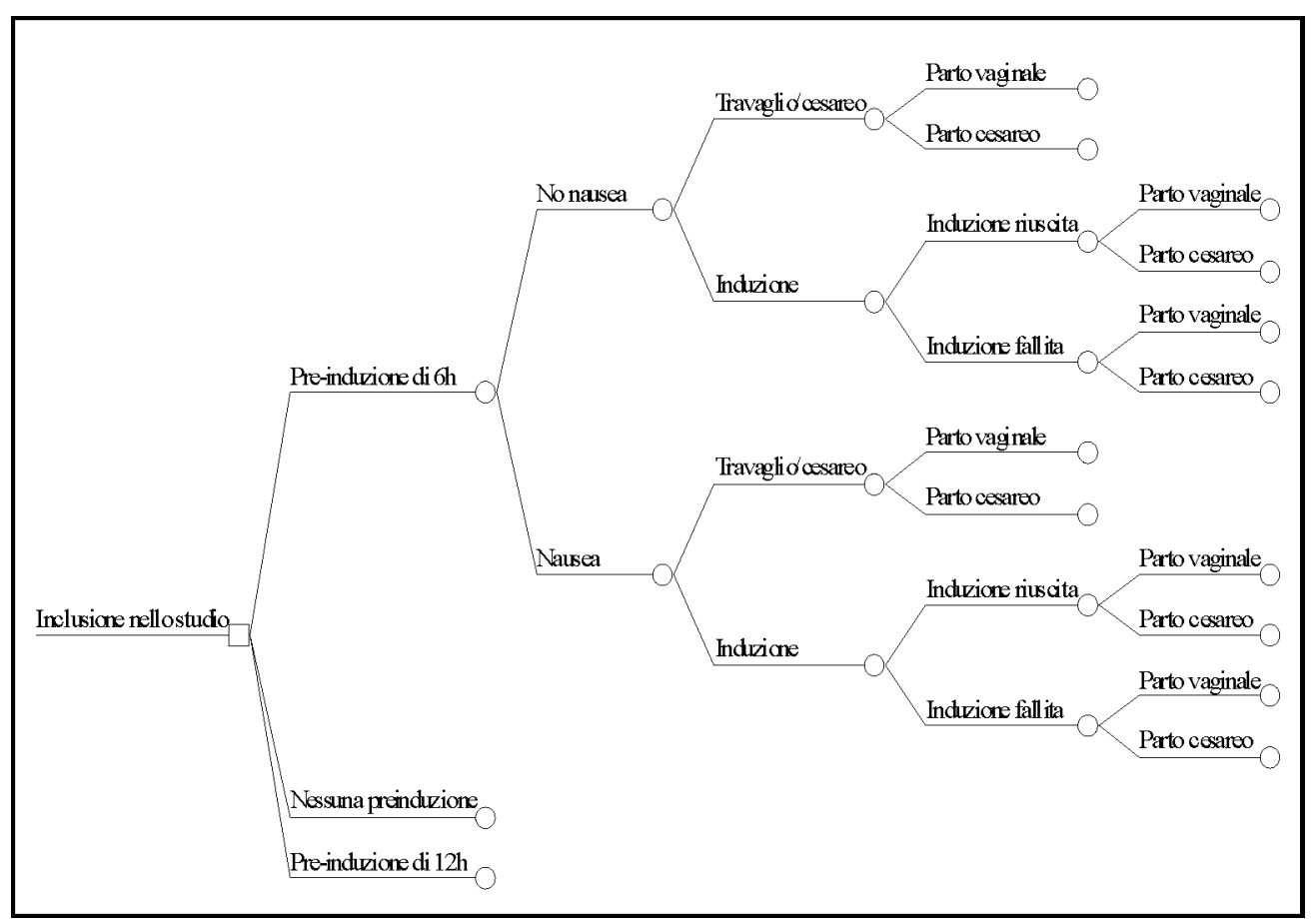


le utilizzato per questo studio rappresenta le tre possibili opzioni terapeutiche successive alla conferma dell'indicazione all'induzione di travaglio in gestanti con cervice immatura:

- pre-induzione con gel di dinoprostone 6 ore prima dell'induzione classica,

- pre-induzione con gel di dinoprostone 12 ore prima dell'induzione e

- nessun trattamento precedente all'induzione.

La figura 1 rappresenta lo sviluppo di uno dei tre rami principali, peraltro identico agli altri due; le probabilità utilizzate per alimentare l'albero sono riportate in tabella 3.

I dati clinici utilizzati per alimentare l'albero decisionale derivano da 3 studi clinici randomizzati, condotti per ottenere la registrazione del gel di dinoprostone (Prepidil®, Pharmacia \& Upjohn) negli USA (11-13), che comprendono un totale di 1731 pazienti con cervice immatura arruolati in 43 centri differenti. I costi analizzati sono quelli marginali, ovverosia i costi aggiuntivi rispetto ad un parto per via vaginale, nella prospettiva dell'ospedale. Gli autori hanno quindi calcolato la media ponderata degli esiti clinici forniti dai tre studi, identificando le seguenti differenze tra i gruppi di donne trattate e quello delle donne indot- te senza pre-trattamento: un minor intervallo di tempo tra l'induzione e il parto, una minor percentuale di parti cesarei, una maggiore prevalenza di effetti collaterali (gastrointestinali e iperstimolazione uterina) e nessuna differenza per quanto riguarda i tracciati cardiotocografici e i punteggi Apgar (valutazione della vitalità del neonato). Dopo la valorizzazione in termini monetari delle varie risorse consumate nella gestione del travaglio di parto, l'analisi ha indicato che la pre-induzione con gel di dinoprostone in donne con indicazione all'induzione e cervice immatura è economicamente vantaggiosa per l'ospedale, essendo associata ad un risparmio per paziente che varia da 159 USD, nel caso di pre-induzione 12 ore prima dell'induzione, a 214 USD (valori del 1993), nel caso il trattamento con dinoprostone avvenga sei ore prima dell'induzione. Il risparmio associato al pre-trattamento con Prepidil ${ }^{\circ}$ era dovuto principalmente alle riduzioni del tempo intercorso tra l'inizio dell'induzione e il parto, del numero di induzioni fallite e del numero di parti cesarei, mentre i maggiori costi dovuti alla maggiore incidenza di effetti indesiderati sono risultati poco influenti sul costo finale. In questo lavoro la robustezza delle stime risultanti dall' analisi decisionale è stata verificata con un'analisi di sensibilità, effettuata mediante l'osservazione delle variazioni del risultato finale indotte da variazioni $( \pm 50 \%)$ dei

\begin{tabular}{|cccc|}
\hline Esito & 6h di pre-induzione & Controllo & 12h di preinduzione \\
\hline No nausea & 0.957 & 0.974 & 0.957 \\
\hline Travaglio/cesareo & 0.465 & 0.056 & 0.465 \\
Parto vaginale & 0.890 & 0.891 & 0.890 \\
Parto cesareo & 0.110 & 0.109 & 0.110 \\
\hline Induzione & 0.535 & 0.944 & 0.535 \\
Induzione riuscita & $\mathbf{0 . 6 4 4}$ & $\mathbf{0 . 5 7 5}$ & $\mathbf{0 . 6 4 4}$ \\
Parto vaginale & 0.822 & 0.866 & 0.822 \\
Parto cesareo & $\mathbf{0 . 1 7 8}$ & $\mathbf{0 . 1 3 4}$ & $\mathbf{0 . 1 7 8}$ \\
Induzione fallita & $\mathbf{0 . 3 5 6}$ & $\mathbf{0 . 4 2 5}$ & $\mathbf{0 . 3 5 6}$ \\
Parto vaginale & 0.822 & 0.866 & 0.822 \\
Parto cesareo & $\mathbf{0 . 1 7 8}$ & $\mathbf{0 . 1 3 4}$ & $\mathbf{0 . 1 7 8}$ \\
\hline Nausea & 0.043 & 0.026 & 0.043 \\
\hline Travaglio/cesareo & 0.465 & 0.056 & 0.465 \\
Parto vaginale & 0.890 & 0.891 & 0.890 \\
Parto cesareo & 0.110 & 0.109 & 0.110 \\
\hline Induzione & 0.535 & 0.944 & 0.535 \\
Induzione riuscita & $\mathbf{0 . 6 4 4}$ & $\mathbf{0 . 5 7 5}$ & $\mathbf{0 . 6 4 4}$ \\
Parto vaginale & 0.822 & 0.866 & 0.822 \\
Parto cesareo & $\mathbf{0 . 1 7 8}$ & $\mathbf{0 . 1 3 4}$ & $\mathbf{0 . 1 7 8}$ \\
Induzione fallita & $\mathbf{0 . 3 5 6}$ & 0.425 & $\mathbf{0 . 3 5 6}$ \\
Parto vaginale & 0.384 & $\mathbf{0 . 4 7 6}$ & $\mathbf{0 . 6 1 6}$ \\
\hline Parto cesareo & $\mathbf{0 . 6 1 6}$ & & \\
\hline
\end{tabular}

Modificata da Hass e Lucas (10)

Tabella 3

Probabilità degli esiti clinici nei tre gruppi di trattamento 
parametri più rilevanti e incerti: in ognuna delle ipotesi testate il pre-trattamento è risultato economicamente più conveniente della rinuncia alla pre-induzione, con risparmi variabili da 43 a 358 USD per paziente trattata

Un'ulteriore conferma dell' attendibilità di queste stime, ritengono gli autori, viene dalla sostanziale analogia col risparmio stimato da Davies e Drummond (14) utilizzando parametri di costo riferiti alla situazione in Gran Bretagna; inoltre, di fronte a misure alternative riguardanti singole voci di costo, sono sempre state utilizzate quelle maggiormente conservative. Pertanto gli autori ritengono che il risparmio stimato sia un obiettivo realisticamente raggiungibile dagli ospedali, indipendentemente dalla quantità di parti gestiti nel reparto di maternità.

Nelle gestanti con reale indicazione all' induzione del travaglio, ma che presentano immaturità cervicale, le procedure d'induzione risultano decisamente piú efficaci se precedute dalla somministrazione di prostaglandine esogene (15). Le vie di somministrazione intravaginale e intracervicale sono le meno invasive e meno gravate di effetti collaterali. Sulla base di questi dati, e osservando che non esistevano dati certi per definire quale tra i vari regimi di somministrazione proposti in letteratura per le prostaglandine risultasse il più vantaggioso, MacKenzie e coll. hanno condotto uno studio clinico controllato (16, 17) per confrontare gli esiti clinici ed economici di due schemi di somministrazione. La sperimentazione, che ha arruolato 955 donne con indicazione all' induzione del travaglio, ha confrontato gli esiti dopo monosomministrazione di $2 \mathrm{mg}$ di gel di dinoprostone con quelli ottenuti ripetendo la somministrazione dopo 6 ore, in caso non fosse insorto il travaglio e il Bishop score fosse ancora $<9$. Entrambi i protocolli prevedevano l'esecuzione dell'induzione con amnioressi e ossitocina qualora il travaglio non fosse insorto 14-20 ore dopo la prima somministrazione di dinoprostone. I risultati della parte clinica della sperimentazione, analizzati separatamente in base alla parità delle pazienti (nullipare e multipare), hanno indicato che la ripetizione della dose di prostaglandina, quando necessaria, riduce le probabilità di dover fare ricorso all'induzione formale con amnioressi e ossitocina in gestanti multipare, ma non nelle nullipare, mentre non ha rilevato alcuna differenza significativa tra gruppi per quanto riguarda gli altri esiti clinici esaminati. La parte di valutazione economica dei due regimi è stata condotta sulla base dei dati raccolti nella cartella clinica, da cui è stato possibile ricavare le quantità di risorse sanitarie consumate da cia-

\section{Figura 2}

Risparmio stimato con due procedure di preinduzione con dinoprostone: sensibilità alle variazioni $( \pm$ $50 \%$ ) dei costi unitari di degenza nel reparto e del taglio cesareo.

Modificata da Hasse Lucas (10)

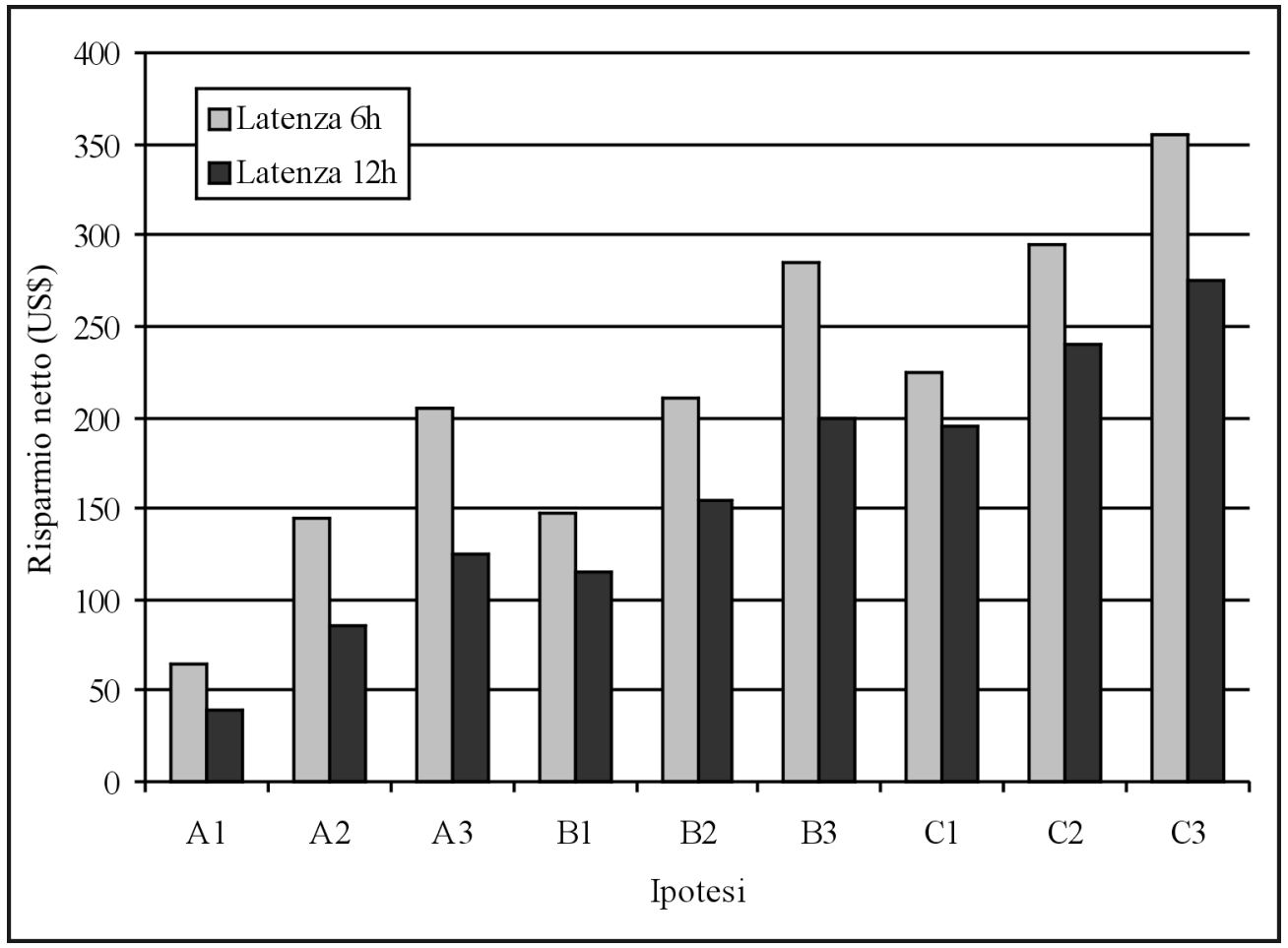

$A=$ riduzione del $50 \%$ del costo-cesareo stimato

$B=$ costo-cesareo stimato

$C=$ aumento del $50 \%$ del costo-cesareo stimato

$1=$ riduzione del $50 \%$ del costo-degenza stimato

$2=$ costo-degenza stimato

$3=$ aumento del $50 \%$ del costo-cesareo stimato. 


\begin{tabular}{|lcccc|}
\hline \multicolumn{1}{|c}{$\begin{array}{c}\text { Spesa media } \\
\text { per paziente }\end{array}$} & Dose singola & Dose ripetuta & \multicolumn{2}{c|}{ Multipare } \\
Dose singola & Dose ripetuta \\
\hline Reparto pre-parto & 81,89 & 97,27 & 76,25 & 79,60 \\
\hline Sala parto & 220,57 & 193,14 & 102,26 & 87,80 \\
\hline Spese pediatriche & 9,93 & 25,22 & 12,19 & 13,90 \\
\hline Reparto post-parto & 352,49 & 344,25 & 319,87 & 321,75 \\
\hline Totale & $\mathbf{6 6 4 , 8 8}$ & $\mathbf{6 5 9 , 8 8}$ & $\mathbf{5 1 0 , 5 7}$ & $\mathbf{5 0 3 , 0 5}$ \\
\hline
\end{tabular}

Modificata da MacKenzie et al (18)

scuna coppia madre-neonato. I costi unitari dei singoli interventi medici sono stati calcolati sulla base delle indicazioni dell' amministrazione ospedaliera, mentre per le tariffe professionali dello staff medico sono state utilizzate le stime di Sculpher et al (18). I risultati dell'analisi economica (tabella 4) hanno indicato che nelle nullipare vi è poca differenza tra i due regimi di somministrazione di dinoprostone per quanto riguarda i costi delle cure perinatali, mentre i costi dovuti alla permanenza nei reparti pre-parto, post-parto e in sala parto sono risultati maggiori con il regime di monosomministrazione; in compenso, questo regime è risultato associato a minori spese per le cure pediatriche del neonato. Nelle pluripare, le principali differenze di costo sono dovute alle minori risorse consumate in sala parto con la strategia della somministrazione ripetuta di dinoprostone in caso di necessità.

I costi medi per paziente della gestione dell'induzione sono risultati inferiori con il regime di doppia somministrazione sia nelle nullipare che nelle pluripare; tuttavia, gli autori avvertono che l'analisi avrebbe potuto favorire il regime di monosomministrazione qualora il disegno dello studio avesse confrontato la seconda dose di dinoprostone, somministrata sei ore dopo la prima, con l'esecuzione di un'induzione con amnioressi e ossitocina in quel momento.

Nel 1991, Davies e Drummond hanno condotto una valutazione farmacoeconomica della gestione del travaglio in Gran Bretagna (14), riferendosi alla prospettiva dell'ospedale o dell' istituzione in cui avviene il parto. In questo lavoro, le pazienti con indicazione all'induzione del travaglio di parto sono state suddivise in due gruppi, a seconda del referto ostetrico preliminare, mantenendo distinte le donne che presentavano una cervice matura (Bishop > 3) da quelle con immaturità cervicale (Bishop <3).

L'analisi é stata condotta con l'utilizzo di due differenti alberi decisionali, uno per gruppo. Quello costruito per analizzare la gestione delle donne con Bishop score < 3 (Figura 3) confrontava le seguenti strategie alternative:

- un periodo di maturazione cervicale stimolata da PGE2, seguita dall'induzione con amnioressi e ossitocina nel caso il travaglio non insorgesse nel periodo di maturazione, e

- l'induzione di travaglio immediata con amnioressi e ossitocina.

L'albero decisionale utilizzato per la valutazione delle alternative terapeutiche nelle donne con Bishop score $>3$ confrontava invece l'induzione con PGE2, eventualmente seguita da amnioressi e ossitocina, con l'opzione di procedere immediatamente all'induzione con amnioressi e ossitocina. Le differenze fondamentali di esito tra le varie strategie alternative consistevano nelle diverse probabilità di dover fare ricorso al taglio cesareo, al parto strumentale e alla trasfusione di sangue per emorragie post-partum molto cospicue. Le probabilità utilizzate per alimentare gli alberi decisionali sono state derivate da tre studi clinici controllati (12, 19-21), provvedendo ad integrare i dati mancanti con i risultati di un' ampia metaanalisi condotta da Keirse (15) e, ove indispensabile, con stime conservative. I costi presi in considerazione dagli autori sono solo quelli sanitari diretti e includono il costo di acquisizione del farmaco, i costi della degenza ospedaliera e quelli del lavoro dei vari componenti lo staff medico. Per quanto riguarda il gruppo delle gestanti con cervice immatura, $i$ risultati dell' analisi decisionale dimostrano che, a fronte di una spesa iniziale nettamente superiore per il gruppo pre-trattato con dinoprostone (59,30 vs. $8,80 £$, sterline inglesi, valore 1989), il costo finale onnicomprensivo di questa strategia terapeutica risultava più economico di circa $50 £$, grazie soprattutto al minor numero di parti cesarei richiesti dopo maturazione cervicale.

Un'analisi di sensibilità condotta su questi risultati, tuttavia, ha indicato che l'ammontare del risparmio ottenibile con le prostaglandine dipende soprattutto dal tempo

\section{Tabella 4}

Costi medi (£) associati ai due regimi $d i$ somministrazione di dinoprostone 
dedicato dallo staff al monitoraggio delle gestanti in attesa del travaglio: assumendo che sia necessario monitorare la paziente pre-indotta con prostaglandine per tutto il periodo precedente l'insorgenza del travaglio di parto, i risparmi si ridurrebbero a circa $6 £$ per paziente. L'analisi decisionale condotta sul gruppo delle donne con indicazione all'induzione e cervice matura ha invece evidenziato che l'induzione con ossitocina è leggermente più conveniente dell'alternativa con PGE2, con un risparmio di circa $£ 17$ per paziente trattata. Gli autori sottolineano che l'analisi è stata condotta in maniera conservativa, a svantaggio delle prostaglandine, e come non abbia preso in considerazione $i$ costi intangibili, legati alla sofferenza per il feto e la madre, che in genere sono minori con le prostaglandine che con l'ossitocina, in quanto la procedura è meno invasiva e il travaglio risulta meno doloroso e medicalizzato. Gli autori, sulla scorta di quanto sostenuto da Donaldson (22), concludono affermando che il tipo di analisi economica adatto a catturare questa componente intangibile sarebbe la "willingness-to-pay", o disponibilità a pagare, ma che siccome già all' analisi dei costi il trattamento con prostaglandine risulta altrettanto o maggiormente conveniente di quello classico, hanno preferito rinunciarvi.

Qualsiasi valutazione economica che intenda comparare l'induzione del travaglio con ossitocina e quella con prostaglandine deve confrontarsi in prima battuta con la notevole differenza nel prezzo d'acquisto tra i due farmaci; uno studio italiano del 1997 (23) ha stimato in 8.080 Lire il costo per paziente indotta con amnioressi e/o ossitocina, a fronte di una spesa di 50.600 Lire per l'induzione con dinoprostone. Una valutazione economica

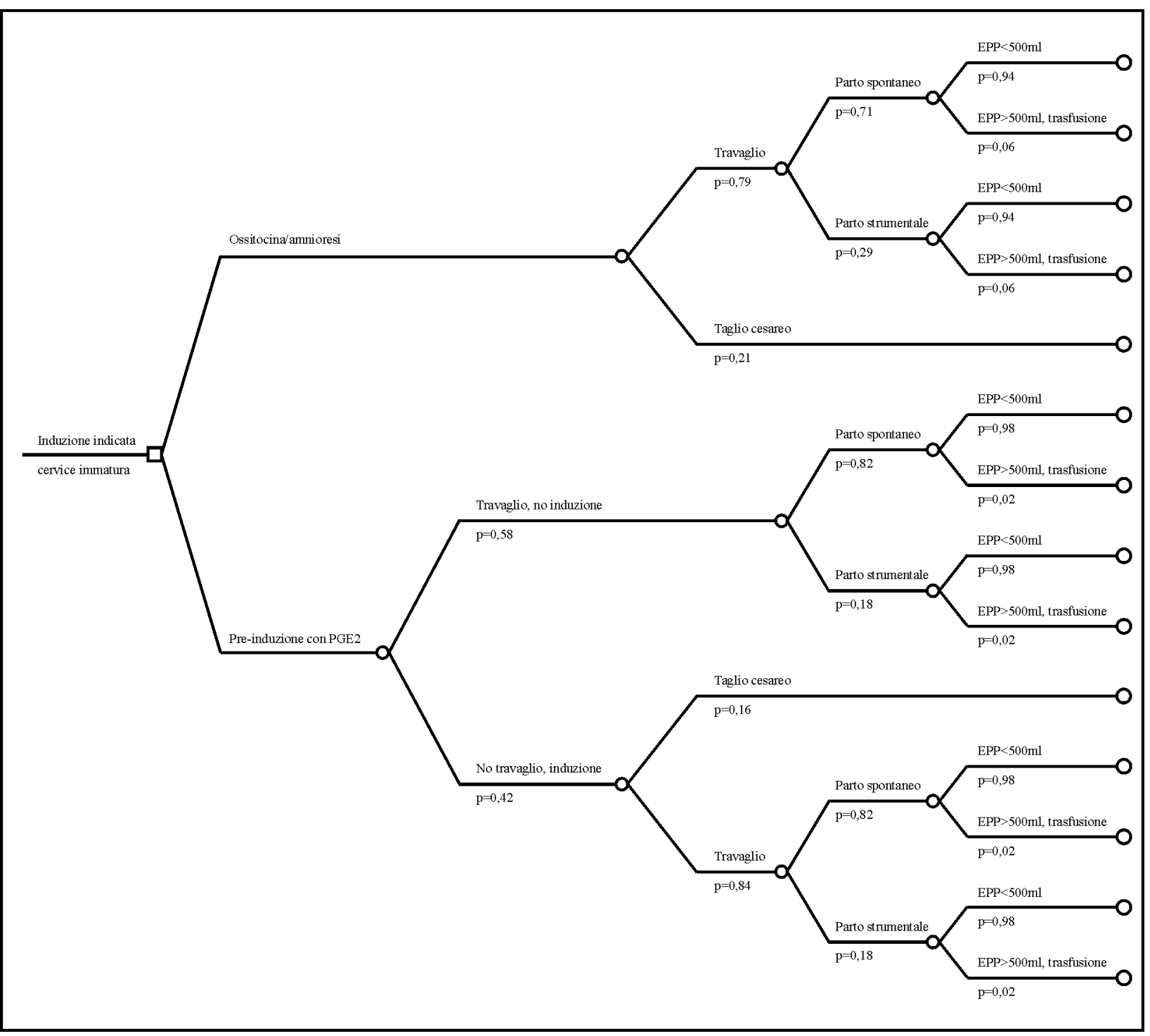

Figura 3

Albero decisionale utilizzato da Davies e Drummond per le gestanti con cervice immatura

$E P P=$ Emorragia post-partum.

Modificata da Davies e Drummond (14) 
completa, tuttavia, deve tenere in considerazione anche le conseguenze, e non solo i costi, di ogni strategia alternativa in competizione. I costi che dovrebbero pertanto essere presi in considerazione comprendono anche i costi della degenza ospedaliera, dell' assistenza medica, infermieristica e ostetrica, gli eventuali costi del parto cesareo, comprendente la sala operatoria, l'anestesista, il chirurgo, e così via.

Numerosi studi clinici mettono in evidenza come l'induzione del travaglio con prostaglandine comporti un minor numero di fallimenti dell'induzione stessa, un minor numero di parti cesarei e una maggiore soddisfazione delle pazienti rispetto all'induzione classica e alla gestione conservativa, di "vigile attesa" (15).

Taylor e Armour (1) hanno pubblicato una rassegna degli studi clinici condotti tra il 1970 ed il 1996 sull'induzione del parto, confrontando gli outcomes dopo prostaglandina E2, eventualmente seguita da amnioressi e/o ossitocina, e quelli ottenuti con l'utilizzo di amnioressi e ossitocina da sole. I dati presi in considerazione si riferivano ad un totale di 3143 pazienti pre-indotte con prostaglandine, confrontate con un gruppo analogo di pazienti che non avevano ricevuto alcun aiuto per promuovere la maturazione cervicale. Questi Autori hanno evidenziato che le donne trattate con dinoprostone avevano una probabilità 7 volte maggiore dei controlli di raggiungere la maturazione cervicale, valutata tramite il Bishop score, maggiori probabilità di insorgenza del travaglio durante la maturazione cervicale e di espletamento del parto entro 12 ore dall'induzione. Inoltre, le donne pre-trattate con prostaglandine avevano minori probabilità di dover fare ricorso all' analgesia epidurale, al parto strumentale e, soprattutto, al parto cesareo. Il lavoro prosegue con una revisione di tre analisi economiche pubblicate nello stesso periodo, tra cui quelle già discusse di Davies e Drummond e Hass e Lucas; secondo Taylor e Armour, nessuna di queste dimostra in maniera robusta ed affidabile il vantaggio economico conseguente alla pre-induzione con dinoprostone, per quanto il complesso dei benefici monetari e intangibili giustifichi il loro uso. Gli autori concludono sottolineando la necessità di ulteriori approfondimenti per poter valutare in maniera affidabile le conseguenze economiche della pre-induzione con prostaglandine e per individuare i gruppi di gestanti che ne traggono maggiore beneficio.

Le conseguenze economiche del ricorso alla somministrazione intravaginale e/o intracervicale di gel di dinoprostone per l'induzione o la pre-induzione del travaglio di parto sono state stimate anche in Italia (23), utilizzando i dati clinici relativi a tutte le pazienti ammesse all'induzione di travaglio nella Clinica Ostetrica e Ginecologica dell'Ospedale San Gerardo di Monza nel periodo gennaio 1987dicembre 1995. Il protocollo terapeutico adottato prevedeva la somministrazione di gel di dinoprostone a tutte le gestanti con indicazione al travaglio e condizioni locali sfavorevoli (cervice immatura, Bishop score < 6), seguita da un periodo di attesa di 6-8 ore. Le donne che in questo periodo di tempo non avevano iniziato il travaglio di parto venivano rivalutate: se il Bishop score risultava ancora inferiore a 6 veniva ripetuta l'applicazione del gel (fino ad un massimo di tre somministrazioni intracervicali o due intravaginali), altrimenti si procedeva all' amnioressi seguita dopo due ore dall'infusione endovenosa di ossitocina.

I risultati dell' analisi dei dati clinici hanno rivelato che, su un totale di oltre 21.000 parti gestiti nel periodo considerato, circa duemila pazienti (9\%), di cui circa 3/4 nullipare, sono state sottoposte alla procedura di induzione con prostaglandine, con una prevalenza totale del $14,7 \%$ di tagli cesarei. Per la valutazione

\begin{tabular}{|lccc|}
\hline \multicolumn{1}{|c}{ Ipotesi } & $\begin{array}{c}\text { Maturazione-induzione } \\
\text { con PGE2 }\end{array}$ & $\begin{array}{c}\text { Induzione con amnioressi } \\
\text { e ossitocina }\end{array}$ & $\begin{array}{c}\text { Differenza di costo } \\
\text { per paziente }\end{array}$ \\
\hline Cervice immatura & & & \\
Scenario base & 167,62 & 220,60 & $-52,98$ \\
Monitoraggio continuo & 247,30 & 253,37 & $-6,07$ \\
\hline Cervice matura & & & \\
Scenario base & 95,18 & 78,20 & $+16,98$ \\
Monitoraggio continuo & 84,84 & 64,42 & $+20,42$ \\
\hline
\end{tabular}

Modificata da Davies and Drummond (14)

\section{Tabella 5}

Costi medi per paziente $(£)$ attesi con le strategie alternative 


\section{Figura 4}

Albero decisionale per la valutazione delle due strategie alternative di induzione

\section{Tabella 6}

Costi unitari conside rati da Zanini e coll.

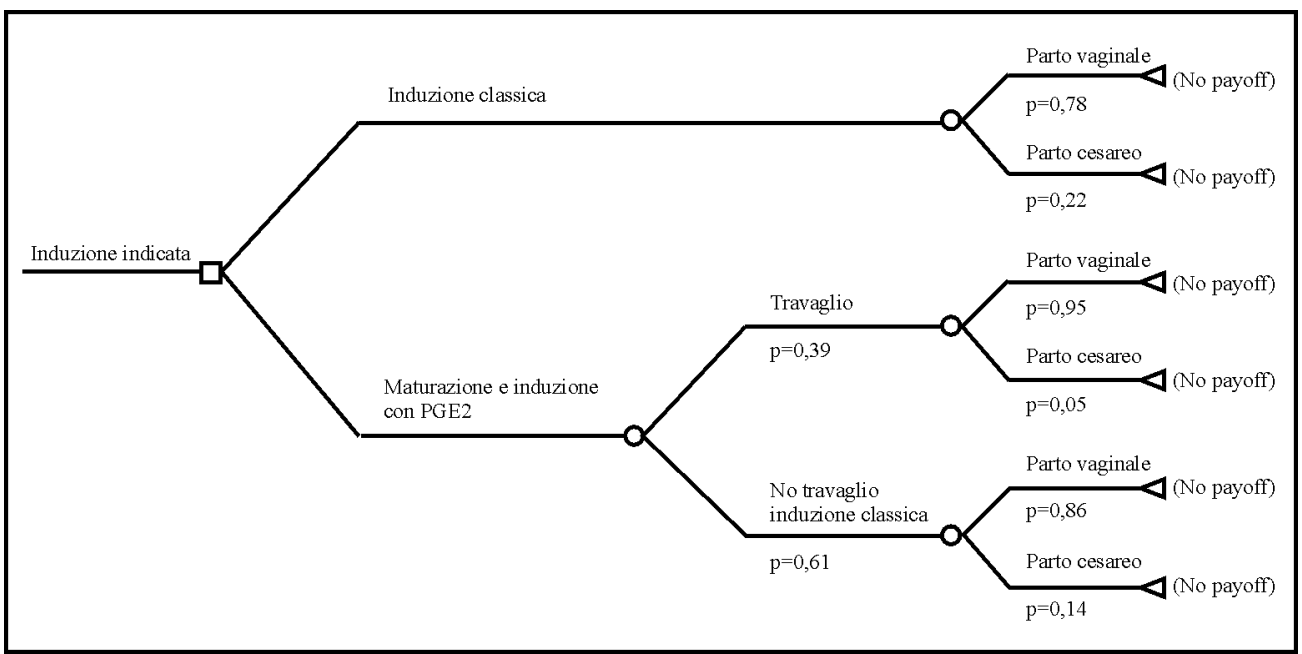

delle implicazioni economiche, gli autori dello studio hanno adottato l'albero decisionale riprodotto in Figura 4, supportato dai dati ricavati da una meta-analisi condotta da Chalmers e coll (24) su studi clinici controllati sull'utilizzo delle prostaglandine esogene in ostetricia.

I costi considerati dall' analisi comprendono la spesa per l'induzione, calcolata sulla base del consumo effettivo osservato, e le risorse consumate per il parto, valorizzate in termini monetari sulla base del tariffario regionale per le prestazioni ospedaliere.

Dopo aver applicato l'albero decisionale di Chalmers e coll. al loro campione e averlo alimentato con i costi unitari riportati in Tabella 6, gli autori hanno calcolato i costi sostenuti dall'ospedale per la gestione dell'induzione con prostaglandine, e li hanno confrontati con quelli che si sarebbero osservati procedendo all'induzione classica. Ne è risultato un risparmio di circa 285 milioni di Lire per il periodo considerato, dovuto al fatto che la gestione con PGE2 del travaglio di parto con condizioni locali sfavorevoli è meno dispendiosa della tecnica classica (1.864.000 vs. 2.010.400), nonostante lo sproporzionato costo iniziale (50.600 vs. 8.080).

Gli autori hanno anche stimato il risparmio potenziale annuo associato all'adozione su scala nazionale del nuovo protocollo d'induzione, assumendo una prevalenza dell'indu- zione del travaglio di parto con condizioni sfavorevoli del $9 \%$, ottenendo un valore di oltre 7 miliardi di Lire, dovuto esclusivamente alla riduzione dei parti cesarei e dei fallimenti dell'induzione, unici parametri considerati.

\section{CONCLUSIONI}

Le prostaglandine esogene vengono utilizzate in ostetricia con il duplice scopo di favorire la maturazione del collo dell'utero e di indurre l'insorgenza del travaglio di parto.

Al momento attuale esse costituiscono l'unica possibilità farmacologica di innescare le modificazioni biochimiche necessarie all'appianamento del collo dell'utero, e pertanto il loro utilizzo è già di per sé giustificato da ragioni cliniche. La revisione delle valutazioni economiche pubblicate sull' argomento ha rilevato che il loro utilizzo è inoltre più costoefficace rispetto alla gestione conservativa con monitoraggi ripetuti, oltre ad essere giudicato più favorevolmente dalle gestanti.

Nonostante il costo di acquisto delle prostaglandine sia molto superiore a quello dell'ossitocina, il loro utilizzo nell'induzione del travaglio di parto ha incontrato un crescente consenso, motivato da giudizi di ordine clinico, come la riduzione delle induzioni fallite e dei conseguenti parti cesarei.

Gli studi farmacoeconomici che confrontano le due alternative indicano che l'induzione

\begin{tabular}{|lcc|}
\hline \multicolumn{1}{|c}{ Prestazione } & Costo Unitario (Lire) & Fonte \\
\hline Induzione con ossitocina/amnioressi & 8.080 & Calcolato \\
\hline Maturazione cervicale e induzione con PGE2 & 50.600 & Calcolato \\
\hline Parto vaginale & 1.632 .000 & Tariffario Regionale \\
\hline Parto cesareo & 3.314 .000 & Tariffario Regionale \\
\hline
\end{tabular}

Tratta da Zanini et al (24) 
del travaglio con dinoprostone è economicamente più vantaggioso nelle donne con indicazione al travaglio e cervice immatura, mentre non consentono di trarre conclusioni definitive per le gestanti con collo dell'utero "maturo", nelle quali la costo-efficacia delle due strategie è comparabile.

Inoltre, nessuno degli studi clinici esaminati ha valutato i benefici economici intangibi- li e difficilmente quantificabili legati all'utilizzo delle prostaglandine per l'induzione di parto, dovuti alla procedura meno invasiva, risultante in un travaglio più fisiologico, meno doloroso e pertanto vissuto in maniera più positiva dalla paziente.

\section{BIBLIOGRAFIA}

1 Taylor SJ and Armour CL. Cost implications in the management of induction of labour. Pharmacoeconomics 1997;12(5):547-54.

2 Taylor AV, Boland JC, MacKenzie IZ. Prostaglandin induced cervical ripening under tocolytic cover in primiparae: results of a double blind placebo controlled trial. Br J Obstet Gynaecol. 1990, 97(9):827-31.

3 Bryman I, Lindblom B, Norstrom A. Extreme sensitivity of cervical musculature to prostaglandin E2 in early pregnancy. Lancet 1982, 2(8313):1471.

4 Wikland M, Lindblom B, Wiqvist N. Myometrial response to prostaglandins during labor. Gynecol Obstet Invest 1984;17(3):131-8

5 Rath W, Osmers R, Adelmann-Grill BC, Stuhlsatz HW, Szevereny M, Kuhn W. Biochemical changes in human cervical connective tissue after intracervical application of prostaglandin E2. Prostaglandins 1993;45(4):375-84.

6 Bishop EH. Pelvic scoring for elective induction. Obstet Gynecol. 1964;24(2):266-8.

7 Goeree R, Hannah M, Hewson S. Cost-effectiveness of induction of labour versus serial antenatal monitoring in the Canadian Multicentre Postterm Pregnancy Trial. CMAJ. 1995, 152(9):1445-50.

8 Hannah ME, Ohlsson A, Farine D, Hewson SA, Hodnett ED, Myhr TL, Wang EE, Weston JA, Willan AR. Induction of labor compared with expectant management for prelabor rupture of the membranes at term. TERMPROM Study Group. NEngl J Med. 1996, 334(16):1005-10.

9 Gafni A, Goeree R, Myhr TL, Hannah ME, Blackhouse G, Willan AR, Weston JA, Wang EE, Hodnett ED, Hewson SA, Farine D, Ohlsson A. Induction of labour versus expectant management for prelabour rupture of the membranes at term: an economic evaluation. TERMPROM Study Group. Term Prelabour Rupture of the Membranes. CMAJ. 1997, 157(11):1519-25.

10 Hass SL, Lucas MJ. Net economic benefit of a manufactured dinoprostone gel for pre-induction cervical ripening. Pharmacoeconomics. 1994, 5(1):39-47.

11 Bernstein P. Prostaglandin E2 gel for cervical ripening and labour induction: a multicentre placebo-controlled trial. CMAJ. 1991,145(10):1249-54.

12 Noah ML, DeCoster JM, Fraser TJ, Orr JD. Preinduction cervical softening with endocervical PGE2 gel. A multicenter trial. Acta Obstet Gynecol Scand. 1987;66(1):3-7.

13 Data on file, The Upjohn Company, Kalamazoo, Michigan, USA.

14 Davies LM and Drummond MF. Management of labour: consumer choice and cost implications. J Obstet Gynaecol 1991, 11 (Suppl.1):S28-S33.

15 Keirse MJNC. Prostaglandins for labour: an overview of controlled data. In: Keirse MJNC, Elder MG, ed. Induction of labour: special issues. Amsterdam: Excerpta Medica, 1991; 3-12.

16 MacKenzie IZ, Burns E. Randomised trial of one versus two doses of prostaglandin E2 for induction of labour: 1 . Clinical outcome. Br J Obstet Gynaecol. 1997, 104(9):1062-7 
17 MacKenzie IZ, Magill P, Burns E. Randomised trial of one versus two doses of prostaglandin E2 for induction of labour: 2. Analysis of cost. Br J Obstet Gynaecol. 1997, 104(9):1068-72.

18 Sculpher MJ, Dwyer N, Byford S, Stirrat GM. Randomised trial comparing hysterectomy and transcervical endometrial resection: effect on health related quality of life and costs two years after surgery. Br $\mathrm{J} \mathrm{Obstet}$ Gynaecol. 1996, 103(2):142-9

19 Kennedy JH, Stewart P, Barlow DH, Hillan E, Calder AA. Induction of labour: a comparison of a single prostaglandin E2 vaginal tablet with amniotomy and intravenous oxytocin. Br J Obstet Gynaecol. 1982, 89(9):704-7.

20 Kennedy JH, Gordon-Wright AP, Stewart P, Calder AA, Elder MG. Induction of labor with a stable-based prostaglandin E2 vaginal tablet. Eur J Obstet Gynecol Reprod Biol. 1982, 13(4):203-8.

21 Cameron A High Bishop score and labour induction. In: The role of prostaglandins in Labour. Wood C., ed., Royal Society of Medicine Services International Congress Series No. 92, London 1985:61-7.

22 Donaldson C, Hundley V, Mapp T. Willingness to pay: a method for measuring preferences for maternity care? Birth. 1998 Mar;25(1):32-9.

23 Zanini C, Folco GC, Limonta Ghezzi G, Caglioni MP, Fenaroli G, Zanini A. Implicazionifarmacoeconomiche nell'induzione del travaglio di parto con prostaglandina E2. Farmacoeconomia 1997, 4(1):31-5.

24 Chalmers I, Enkin M, Keirse MJNC. Effective care in pregnancy and childbirth. 1991; Vol.2: 1015-25. 